
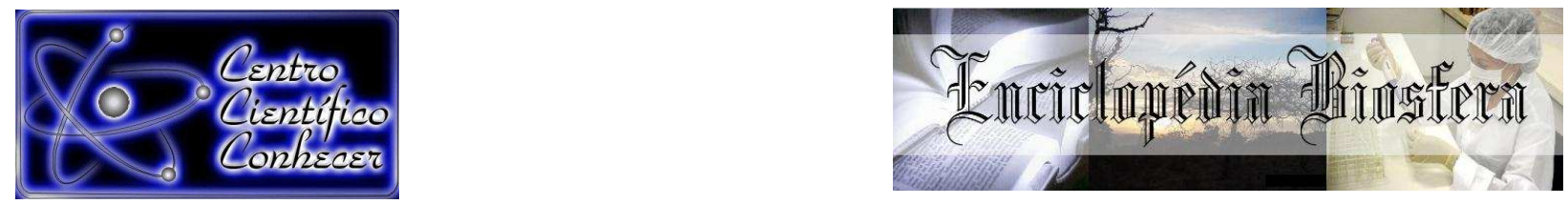

\title{
AVALIAÇÃO DE DOIS TESTES DE COMPORTAMENTO HIGIÊNICO EM COLÔNIAS DE Apis mellifera
}

Marcelo da Silveira Meirelles Pinheiro ${ }^{1}$, Ana Flávia Basso Royer ${ }^{2}$, Osvaldo Martins de Souza ${ }^{3}$, Mérik Rocha Silva ${ }^{4}$.

1 Doutorando Universidade de São Paulo (USP). Faculdade de Zootecnia e Engenharia de Alimentos (FZEA), Pirassununga, SP. marcelosmpinheiro@usp.br 2 Docente Instituto Federal de Educação, Ciência e Tecnologia de Mato Grosso do Sul (IFSM), Nova Andradina, MS.

3 Docente Universidade do Estado de Mato Grosso (UNEMAT), Pontes e Lacerda, MT.

4 Docente Instituto Federal de Educação, Ciência e Tecnologia de Mato Grosso (IFMT), Pontes e Lacerda, MT.

Recebido em: 08/09/2015 - Aprovado em: 14/11/2015 - Publicado em: 01/12/2015 DOI: http://dx.doi.org/10.18677/Enciclopedia_Biosfera_2015_009

\section{RESUMO}

O comportamento higiênico é uma ferramenta essencial no melhoramento em enxames. Diferentes testes avaliam o comportamento higiênico, os mais utilizados são o teste de congelamento e o de perfuração de crias operculadas, sendo o segundo o mais usado por técnicos no campo. Porém os procedimentos e a aplicabilidade desse teste por apicultores ainda requer um conhecimento prévio de cálculos matemáticos e demanda mínima de 15 a 16 dias para repetição. Devido as estes fatores o objetivo deste trabalho foi de avaliar e comparar dois testes de comportamento higiênico, buscando uma forma simplificada mais prática e rápida. Os dados revelaram não haver diferença significativa entre os tratamentos (primeiro ano com o fator de correção e segundo ano sem o fator de correção) $(p<0,05)$. 0 desempenho médio individual de cada grupo apresentou valores diferenciados 0 grupo $D$ teve diferença significativa aos demais com média de $91,55 \%$ superior ao grupo B com média $88,28 \%$ que também teve diferença significativa aos demais grupos. Os grupos E, A e C não tiveram diferença significativa com médias $86,11 \%$, $85,30 \%$ e $84,78 \%$. O teste do comportamento higiênico mais simplificado sem o fator de correção foi estatisticamente semelhante ao teste com fator de correção, podendo ser utilizado pelos apicultores na seleção de colmeias para o comportamento higiênico.

PALAVRAS-CHAVE: Desempenho, manejo, métodos.

\section{EVALUATION OF TWO BEHAVIOUR HYGIENIC TESTS IN Apis mellifera COLONY}

\section{ABSTRACT}

The hygienic behavior is an essential tool in the improvement in swarms. Different tests measure hygienic behavior, the most used are freezing test and the offspring of operculated drilling, the second most used by technicians in the field. But the 
procedures and the applicability of this test for beekeepers still requires a prior knowledge of mathematical calculations and minimum demand of 15 to 16 days to repeat. Due to these factors the aim of this study was to evaluate and compare two hygienic behavior tests, seeking a simplified most practical and expeditious manner. The data showed no significant difference between treatments (first year with the correction factor and second year without correction factor) ( $p<0.05)$. The average individual performance of each group had different values $D$ group had significant difference to other averaging $91.55 \%$ higher than the group B with an average $88.28 \%$ also had significant difference with the other groups. Groups E, A and C had no significant difference with average $86.11 \%, 85.30 \%$ and $84.78 \%$. The test more streamlined hygienic behavior without the correction factor was statistically similar to the test with correction factor and can be used by beekeepers in hives selection for hygienic behavior.

KEYWORDS: Performance, management, methods

\section{INTRODUÇÃO}

As abelhas africanizadas brasileiras têm geneticamente resistência a doenças e a parasitas atribuídas ao comportamento higiênico. Este comportamento se traduz na habilidade natural da abelha em detectar e remover crias mortas, doentes ou infestadas por ácaros da colmeia, bem como também remover material estranho de dentro das colmeias permitindo existir colônias mais sadias, mais populosas e melhores produtoras de mel (ROTHENBUHLER, 1964; GRAMACHO, 1999; OLINTO, 2014).

O comportamento higiênico é uma forma alternativa de controle ao uso de produtos quimioterápicos, por esse motivo, constitui-se ferramenta essencial na seleção e melhoramento em enxames de pequenos apiários com 100 colmeias até apiários maiores superiores a 500 colmeias (ROTHENBUHLER, 1964; GRAMACHO, 1999). Porém, mesmo sendo mais eficientes neste processo, a prevalência e a frequência dos genes contribuintes ao comportamento nos enxames encontram-se em menor escala fazendo necessário a seleção e multiplicação (GRAMACHO \& GONÇALVES, 1994).

No comportamento higiênico das abelhas africanizadas são definidas algumas etapas como a inspeção, a desoperculação e por fim a remoção da cria morta (PALACIO et al., 2010). Para que uma colmeia seja considerada como higiênica ou não higiênica é necessário fazer um estudo com base em testes na capacidade de limpeza das crias mortas ou doentes no prazo de 24 horas, neste caso os enxames que conseguirem remover $80 \%$ ou mais das crias mortas são consideradas como higiênicas e as outras abaixo desse valor poderão ser descartadas sendo determinadas como não higiênicas (GRAMACHO \& GONÇALVES, 1994).

Após o acasalamento da rainha os espermatozoides são armazenados na espermateca, o deslocamento dos aglomerados de espermatozoides da espermateca até a entrada do oviduto central para a fecundação dos óvulos varia entre 15 a 16 dias, para evitar erro nos dados de coleta do comportamento higiênico, recomenda-se repetir no mínimo três vezes o teste pelo mesmo período de tempo (DELANEY et al., 2011; TÜRUP et al., 2013; HATJINA et al., 2014;).

Existem vários artigos com diferentes testes de estudo para avaliar o comportamento higiênico, os mais utilizados, são o teste de congelamento de crias e o teste de perfuração de crias operculadas (GONÇALVES \& KERR, 1970; NEWTON \& OSTASIEWSKI, 1986), entretanto o segundo método já teve modificações sendo 
considerado o mais usado por técnicos no campo (GRAMACHO \& GONÇALVES, 1994).

Com o advento do início do projeto APIS - Apicultura Integrada e Sustentável iniciado em 2003, a apicultura no Estado de Mato Grosso teve uma maior adesão de novos apicultores e um enorme crescimento de apiários. O processo de especialização profissional e a busca no aumento da produção de mel fez-se necessário avaliar o desempenho produtivo das abelhas para uma eventual seleção e acompanhamento para o melhoramento genético. Porém os procedimentos e a aplicabilidade desse teste por apicultores ainda requer um conhecimento prévio de cálculos matemáticos e demanda mínima de 15 a 16 dias para repetição. Devido as estes fatores o objetivo deste trabalho foi avaliar e comparar dois testes de comportamento higiênico em colônias de Apis mellifera, buscando uma forma simplificada do teste que seja mais prática e rápida.

\section{MATERIAL E MÉTODOS}

O experimento foi conduzido no município de Pontes e Lacerda no apiário localizado no campus da UNEMAT - Universidade do Estado de Mato Grosso. Todas as colmeias eram do tipo padrão Langstroth e tinham no mínimo quatro quadros com crias operculadas.

Utilizou-se 25 colmeias da região do Vale do Guaporé, localizado a Oeste do Estado de Mato Grosso que compreende desde a cidade de Cáceres até a cidade de Comodoro. Foram formados cinco grupos de enxames da região do Vale do Guaporé: $A$ = Região de Nova Lacerda; $B$ = Região Conquista D'Oeste Serrado; $C$ = Região Conquista D'Oeste Altitude (700 a 1700 m); $D=$ UNEMAT e $E$ = Região Pontes e Lacerda.

O período de coleta iniciou-se no início da seca de cada ano (abril 2008-2009) onde há baixo fluxo de néctar para evitar a influência do meio ambiente sobre o comportamento. O delineamento experimental foi inteiramente casualizado com dois tratamentos e seis amostras repetidas no tempo. Foram realizados dois testes de comportamento higiênico, um adaptado e proposto por GRAMACHO (1999) e o outro foi o mesmo teste, porém sem o fator de correção e em menor tempo.

No primeiro experimento (1), os dados coletados de seis repetições no tempo (primeiro ano) foram espaçados em 20 dias para cada coleta, a técnica consistiu na escolha de um quadro central de favo novo com crias operculadas (10 a 14 dias de idade) (GRAMACHO, 1999), neste quadro foi delimitado duas áreas vizinhas em forma de losango cada uma com 100 células operculadas (10 linhas por 10 fileiras) a primeira área foi destinada para perfuração e a segunda área como testemunha sem perfuração.

A perfuração das crias operculadas foi feita com alfinete entomológico introduzido no centro do opérculo, após a perfuração esperou-se 24 horas pelo trabalho das operárias higiênicas na desoperculação e remoção das crias mortas ou danificadas pelo alfinete, importante salientar que no teste do comportamento higiênico o tempo de observação das $12 \mathrm{~h}$ a $48 \mathrm{~h}$ pós-perfuração das crias pode superestimar as médias, diferenciando estatisticamente (SANDOZ, 2011), porém em horários diferentes (24h, 48h e 72h) não apresentam diferença (FREY, 2014).

$\mathrm{Na}$ sequência foram contadas as células vazias nas duas áreas e realizados os cálculos para determinar a taxa de limpeza na área perfurada e na área testemunha que posteriormente foram submetidos a um fator de correção "Z" estimando por diferença a porcentagem real (GRAMACHO \& GONÇALVES, 1994; GRAMACHO, 1999). 
No segundo experimento (2), os dados coletados de seis repetições no tempo (segundo ano) foram espaçados em 10 dias para cada coleta com a mesma técnica anterior, porém a porcentagem real foi calculada direta sem os cálculos do fator de correção. Os dados foram analisados pelo pacote estatístico SISVAR.

\section{RESULTADOS E DISCUSSÃO}

De acordo com análise estatística, $(p<0,05)$, não houve diferença significativa entre os tratamentos (primeiro ano com o fator de correção e segundo ano sem o fator de correção) para todas as colmeias analisadas (Tabela 1), o tratamento mais simples (sem o fator de correção) detectou numericamente um valor maior do comportamento higiênico na metade do tempo (10 dias). Em outra pesquisa também com perfuração de crias avaliando o comportamento higiênico de comeias e sua primeira geração ( $\mathrm{F} 1)$, apresentou variação de 64 a $88 \%$, usando a mesma técnica anterior com o fator de correção (PICKLER, 2009).

BUGALHO (2009) em um experimento trabalhando com comportamento higiênico por perfuração de crias e observando antes, durante e após a chuva o autor obteve como média geral no teste variação de $69,27 \%$ a $72,26 \%$. No sertão paraibano trabalhando com cinco diferentes apiários em experimento com mesma técnica de perfuração de crias, o percentual do comportamento higiênico foi semelhante em Condado (93,96\%), Pombal (94,30\%), Jericó $(87,63 \%)$ e São Domingos (95,20\%), sendo que no apiário de São Bentinho a média de 76,31\% diferenciou-se das demais (OLINTO, 2014). Em outra região, no sudeste brasileiro, analisando o comportamento higiênico em diferentes cidades foi constatado valor médio de $98,6 \%$ para a cidade de Taubaté e valor médio de $57,7 \%$ para a cidade de Viçosa, os autores explicam que esta variação se deve a fatores populacionais, vigor da colônia e de fatores ainda desconhecidos (PINTO et al., 2012).

TABELA 1. Resultado das médias gerais do método de perfuração das células de crias com o fator de correção e sem fator de correção.

\begin{tabular}{cccc}
\hline Tratamentos & Média & Teste Tukey & Erro padrão \\
\hline 1 & $86,97 \%$ & a & $0,55 \%$ \\
\hline 2 & $87,44 \%$ & a & \\
\hline
\end{tabular}

Médias seguidas de mesma letra minúscula na coluna não diferem pelo Teste Tukey no nível de $5 \%$ de probabilidade.

O desempenho geral de cada grupo ao comportamento higiênico também foi avaliado nos dois anos, não apresentando diferença significativa pelo teste de Tukey $(p<0,05)$ (Tabela 2).

TABELA 2. Resultado das médias gerais de cada grupo por ano.

\begin{tabular}{cccccc}
\hline Grupos & $\mathbf{A}$ & $\mathbf{B}$ & $\mathbf{C}$ & $\mathbf{D}$ & $\mathbf{E}$ \\
\hline Média 1 & $85,87 \%$ & $88,53 \%$ & $84,93 \%$ & $92,10 \%$ & $87,40 \%$ \\
Média 2 & $84,73 \%$ & $88,03 \%$ & $84,63 \%$ & $91,00 \%$ & $86,83 \%$ \\
Teste & $\mathrm{a}$ & $\mathrm{a}$ & $\mathrm{a}$ & $\mathrm{a}$ & $\mathrm{a}$ \\
Tukey & & & & & \\
Erro & & & $1,12 \%$ & & \\
padrão & & & & & \\
\hline
\end{tabular}

de $5 \%$ de probabilidade. 
Em relação ao comportamento higiênico no desempenho médio individual dos dois anos de cada grupo, segundo o Teste Scott-Knott, o grupo $D$ teve diferença significativa aos demais apresentando uma média de $91,55 \%$ superior ao grupo B com média $88,28 \%$ que também teve diferença significativa aos demais grupos. Os grupos E, A e C não tiveram diferença significativa com médias $86,11 \%, 85,30 \%$ e $84,78 \%$, respectivamente (Tabela 3 ).

TABELA 3. Média individual do desempenho dos grupos.

\begin{tabular}{cccc}
\hline Grupos & Médias & Teste Scott-Knott & Erro Padrão \\
\hline A & $85.30 \%$ & C & \\
B & $88.28 \%$ & b & \\
C & $84.78 \%$ & C & $0,81 \%$ \\
D & $91.55 \%$ & a & \\
E & $86.12 \%$ & C & \\
\hline
\end{tabular}

Médias seguidas de mesma letra minúscula na coluna não diferem pelo Teste Scott-Knott.

Para GRAMACHO \& GONÇALVES (1994) uma colônia higiênica é aquela que remove $80 \%$ ou mais de crias perfuradas, sendo assim, todos os grupos apresentaram um bom comportamento higiênico independente do método usado ou do ano avaliado.

\section{CONCLUSÕES}

O desempenho geral de cada grupo quanto ao comportamento higiênico não apresentou diferença significativa nos dois anos. O teste do comportamento higiênico mais simplificado de perfuração das células de crias sem o fator de correção não apresentou diferença estatística ao teste com fator de correção, podendo ser uma alternativa mais prática e rápida a ser utilizada pelos apicultores na seleção de colmeias para o comportamento higiênico.

\section{AGRADECIMENTOS}

Em especial aos alunos da UNEMAT - Pontes e Lacerda que contribuíram na coleta de dados e aos apicultores da FEAPISMAT da cidade de Conquista D'Oeste pela doação de parte dos enxames.

\section{REFERÊNCIAS}

BUGALHO, V. A. Influência das precipitações pluviométricas e da atividade forrageira das abelhas africanizadas (Apis mellifera L.) no comportamento higiênico. Dissertação de mestrado apresentada a Faculdade de Filosofia, Ciências e Letras de Ribeirão Preto - USP. 108p. 2009.

DELANEY, D. A., KELLER, J. J., CAREN, J. R. \& TARPY, D. R.. The physical, insemination, and reproductive quality of honey bee queens (Apis mellifera L.). Apidologie, 42(1), 13 p. 2011.

FREY, E. Der Einfluss von Wirtsfaktoren der Honigbiene (Apis mellifera L.) auf den Reproduktionserfolg der parasitischen Milbe Varroa destructor (Anderson ENCICLOPÉDIA BIOSFERA, Centro Científico Conhecer - Goiânia, v.11 n.22; p.2911 2015 
\& Trueman) auf die Auswirkungen einer horizontalen Verbreitung des Parasiten auf den Befall der Bienenvölker. Dissertation zur Erlangung des Grades eines Doktors der Allgemeinen Agrarwissenschaften. Landesanstalt für Bienenkunde der Universität Hoh Enheim. 66 p. 2014.

GONÇALVES, L. S.; KERR, W. E. Genética, Seleção e Melhoramento. 1. Noções sobre genética e melhoramento em abelhas. In: CONGRESSO BRASILEIRO DE APICULTURA, 1., Florianópolis, 1970. Anais. p. 8-36. 1970.

GRAMACHO, K. P. Fatores que interferem no comportamento higiênico das abelhas Apis mellifera. Tese de Doutorado apresentada a FFCLRP-USP, Ribeirão Preto, 225 p. 1999.

GRAMACHO, K. P.; GONÇALVES, L. S. Estudo comparativo dos métodos de congelamento e perfuração de crias para avaliação do comportamento higiênico em abelhas africanizadas. In: CONGRESSO LATINOIBEROAMERICANO DE APICULTURA, 4. 1994. Anais. Cordoba-Argentina. p.45. 1994.

HATJINA, F, BIEŃKOWSKA, M., CHARISTOS, L., CHLEBO, R., COSTA, C, DRAŽIĆ, M. M., FILIPI, J., GREGORC, A, IVANOVA, N. E, KEZIĆ, N., KOPERNICKY, J., KRYGER, P, LODESANI, M., LOKAR, V., MLADENOVIC, M, PANASIUK, B., PETROV, P. P., RAŠIĆ, S., SKER, M. I. S., VEJSNÆS, F and WILDE, J. A review of methods used in some European countries for assessing the quality of honey bee queens through their physical characters and the performance of their colonies. Journal of Apicultural Research 53 (3): 337-363. 27 p. 2014.

NEWTON, D. C., OSTASIEWSKI N. J. A simplified bioassay for beliavioral resistance to American Foulbrood in honey bees (Apis mellifera L.). American Bee Journal,v. 126, n. 4, p. 278-281,1986.

OLINTO, F. A. Comportamento higiênico e identificação de patógenos em colmeias de Apis mellifera L. Africanizadas no sertão paraibano. Tese de Mestrado apresentada UFCG. Pombal-PB. 62 p. 2014.

PALACIO, M.A., RODRIGUEZ, E., GONCALVES, L., BEDASCARRASBURE, E., SPIVAK, M. Hygienic behaviors of honey bees in response to brood experimentally pin-killed or infected with Ascosphaera apis. Apidologie. 41(1). p 602-612. 2010.

PICKLER, M.A. Defensividade, higiene, produção de própolis e mel com duas gerações de A. mellifera. Dissertação (Mestrado em Zootecnia) - Campus de Marechal Candido Rondon, Universidade Estadual do Oeste do Paraná, Marechal Rondon, 59 p. 2009.

PINTO, F. A; PUKER, A; BARRETO, L.M.R.C.; MESSAGE, D. The ectoparasite mite Varroa destructor Anderson and Trueman in southeastern Brazil apiaries: effects of the hygienic behavior of Africanized honey bees on infestation rates. Arq. Bras. Med. Vet. Zootec., v.64, n.5, p.1194-199, 2012.

ROTHENBUHLER, W. C. Behaviour genetics of nest cleaning in honey bees. IV. Responses of $\mathrm{F} 1$ and backcross generations to disease-killer brood. American 
Zoology, v.4, p.111-123, 1964.

SANDOZ, J. C. Behavioral and neurophysiological study of olfactory perception and learning in honeybees. Frontiers in Systems Neuroscience, v. 5, n. 98, p. 1-20, 2011.

STÜRUP, M., BAER-IMHOOF, B., NASH, D.R., BOOMSMA, J.J., BAER, B. When every sperm counts: factors affecting male fertility in the honeybee Apis mellifera. Behavioral Ecology. 24. p 1192-1198. 2013. 